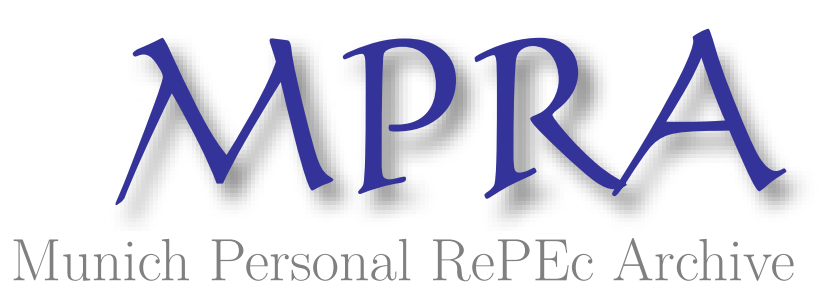

Social choice and game theory: recent results with a topological approach

Chichilnisky, Graciela

1983

Online at https://mpra.ub.uni-muenchen.de/8059/

MPRA Paper No. 8059, posted 03 Apr 2008 04:37 UTC 


\title{
Social choice and game theory: Recent results with a topological approach
}

\author{
G. CHICHILNISKY* \\ University of Essex and Columbia University
}

\section{Introduction}

This chapter presents a summary of recent results obtained in game and social choice theories, and highlights the application and the development of tools in algebraic topology. The purpose is expository: no attempt is made to provide complete proofs, for which references are given, nor to review the previous work in this area, which covers a significant subset of the economic literature.

The aim is to provide an oriented guide to recent results, through economic examples with geometric interpretations, and to indicate possibly fruitful avenues of research.

The use of topological tools has a long tradition in economic analysis, which goes back to the work of Von Neumann on balanced economic growth of 1937 and 1945 . He proved a generalization of Brouwer's fixed point theorem that was the basis of Kakutani's theorem. In game theory and in general equilibrium market analysis, fixed point methods are the topological methods most frequently utilized to show existence of solutions. As a matter of fact, fixed point theorems are the largest part of applications of topology to economics as a whole. Instead of topological methods, social choice theory has traditionally been formulated in a

* This research was supported partly by NFS Grant SES 7914050 . I thank G. Heal, C. Henry, J.M. Lasry, and M. Vergne for comments and suggestions. A French version of this chapter appears in E. Malinvaud, ed., Cahiers du Seminaire d'économetrie (CNRS, Paris,1982). 
combinatorial fashion, following the first formal works of Arrow (1951) and Black (1948) in this area.

However, we shall now show that many problems of game and social choice theories, when properly formulated, exhibit an intrinsic topological structure that may be fruitfully examined with algebraic topology tools that go beyond fixed point theorems, such as homotopy and cohomology theories. This allows us to tap a wealth of existing topological techniques, as well as to develop new ones, to resolve problems in social choice and game theories.

This chapter studies certain social choice paradoxes and their resolution, the relation of a fixed point theorem with the social choice paradox, the equivalence of the Pareto condition and the existence of a dictator, majority rules, aggregation in large economies, and the aggregation of Von Neumann-Morgenstern utilities for choices under uncertainty.

Within game theory, we summarize results on the manipulation of games, the existence of Nash equilibrium of certain non-convex games and the fairness of these games, and the existence and characterization of strategy-proof games, a problem that appears in the literature on economies with public goods.

\section{Social choice}

Social choice theory is concerned with providing a rationale for collective decisions when individuals have diverse opinions. Voting is an obvious way in which societies aggregate individual preferences into collective preferences. It has been known for a long time that majority voting may be in contradiction with certain basic criteria of rationality of preferences, such as the transitivity of the social choice; this phenomenon is usually called the "Condorcet" effect; Condorcet first formalized it in 1785 in a book on the theory of elections. The general theory of elections became a fertile field of research following the work of Black in 1948 and Arrow in 1951. Arrow stated formally a set of apparently reasonable criteria required for the aggregation of individual preferences, and proved that they are inconsistent.

One way of formalizing the problem is as follows: a voting procedure typically takes into account only the relative value that the individuals assign to different choices (i.e. ordinal preferences), rather than the intensity with which they prefer one choice to another (e.g. preferences derived from utilities). This is one source of the social choice paradox, because, as we shall see, spaces of ordinal preference are rather different from spaces of utility functions. Utility functions are real-valued functions and as such can be aggregated or summed. Spaces of utility functions are linear, and, in particular, topologically trivial or contrac- 
tible. ${ }^{1}$ Instead, spaces of ordinal utilities are not linear, they cannot be deformed into linear spaces, and furthermore they are not topologically trivial. This will be seen to be the source of the social choice paradox because contractible spaces, and only them, admit appropriate aggregation rules (see theorem 10).

We now need some definitions.

The social choice problems studied here are similar to procedures of selection of a vector of public goods, and therefore lend themselves naturally to representation in Euclidean space. Let $X$ be a space of alternatives or choices, contained in the positive orthant of Euclidean space $R^{n}$, i.e. $R^{n+}$. Assume $X$ is a cube in $R^{n+}$. One can also consider more general cases; however, since the results are topological, they will be automatically valid for continuous deformations of all objects under consideration.

We use here the notation of Chichilnisky (1980d) throughout, and the reader is referred to it. A preference $p$ is a $C^{1}$ vector field over the space of alternatives, which is locally integrable, see Debreu (1972). This means that to each alternative $x$ one attaches a vector $p(x) \in R^{n}$ (in a continuously differentiable fashion) which indicates a gradient or the direction of the largest increase of utility, i.e. the normal to an indifference surface of an utility. Since we are considering here ordinal preferences, it is the direction rather than the length of the vectors that matters. Therefore, we normalize the vectors at each point to be of length 1 , i.e. $\|p(x)\|=1 \forall x$ in $X$ (see fig. 6.1). $P$ represents the space of preferences over $X$.

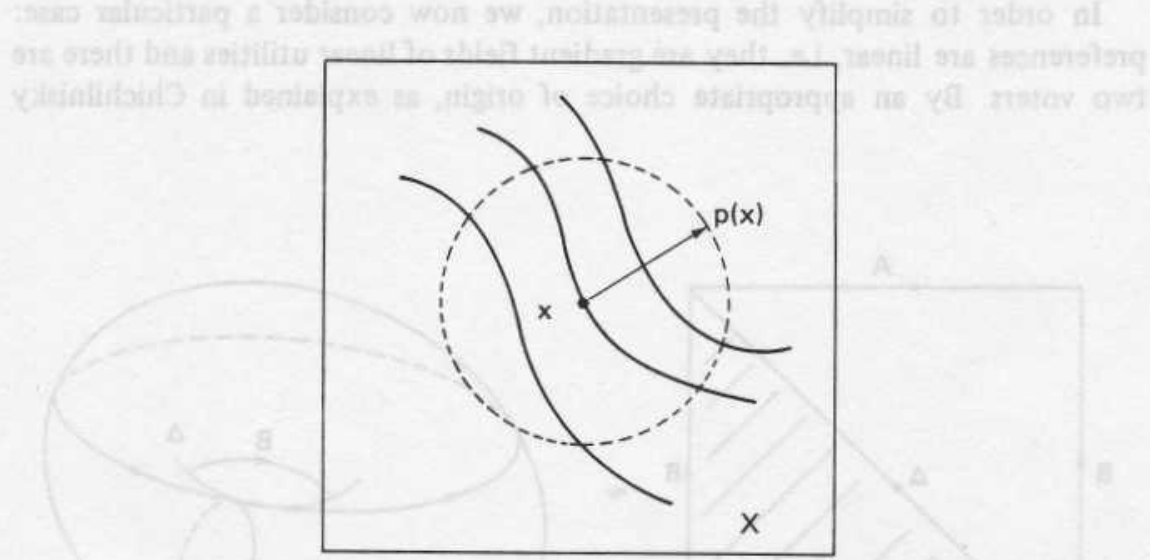

Figure 6.1. A preference over $R^{2}: p(x)$ is the gradient vector at a choice $x$.

1 A topological space $X$ is topologically trivial or contractible when it admits a continuous deformation through itself into one of its points. It is therefore topologically equivalent to a point. Formally, $X$ is contractible if there exists a continuous map $f: X \times[0,1]$ $\rightarrow X$ such that $f(x, 0)=x \forall x$ in $X$, and $f(x, 1)=x_{0}$, all $x$ in $X$, some $x_{0}$ in $X$. 
With $k$ individuals, a social choice rule $\phi$ is thus a map that associates a collective preference to each $k$-tuple of individual preferences, i.e.

$$
\phi: P^{k} \rightarrow P \text {. }
$$

$P^{k}$ is called the space of profiles of individual preferences. The continuity of $\phi$ is defined according to the usual topology for vector fields, implying that proximity of preferences in $P$ is equivalent to the proximity of their indifference surfaces. Anonymity is defined by the condition $\phi\left(p_{1}, \ldots, p_{k}\right)=\phi\left(p_{n}, \ldots, p_{n_{k}}\right)$, where $n$ is any permutation of the set of integers $\{1, \ldots, k\}$. Respect of unanimity means that, whenever all the individuals have identical preference (for all possible choices), then the outcome has the same preference as well, i.e.

$$
\phi=(p, \ldots, p)=p, \quad \forall p \text { in } P .
$$

$\phi$ is Pareto if the outcome $\phi\left(p_{1}, \ldots, p_{k}\right)$ prefers choice $x$ to $y$ whenever all preferences in the profile $\left(p_{1}, \ldots, p_{k}\right)$ prefer $x$ to $y$. Note that respect of unanimity is a much weaker condition than Pareto, since it is only binding when all individuals have identical preferences for all choices. $\phi$ is dictatorial if $\phi\left(p_{1}, \ldots, p_{k}\right) \equiv p_{d}$ for some $d \in\{1, \ldots, k\}$, for all profiles $\left(p_{1}, \ldots, p_{k}\right)$ in $P^{k}$. Note that anonymity is a stronger condition than non-dictatorship.

In order to simplify the presentation, we now consider a particular case: preferences are linear, i.e. they are gradient fields of linear utilities and there are two voters. By an appropriate choice of origin, as explained in Chichilnisky

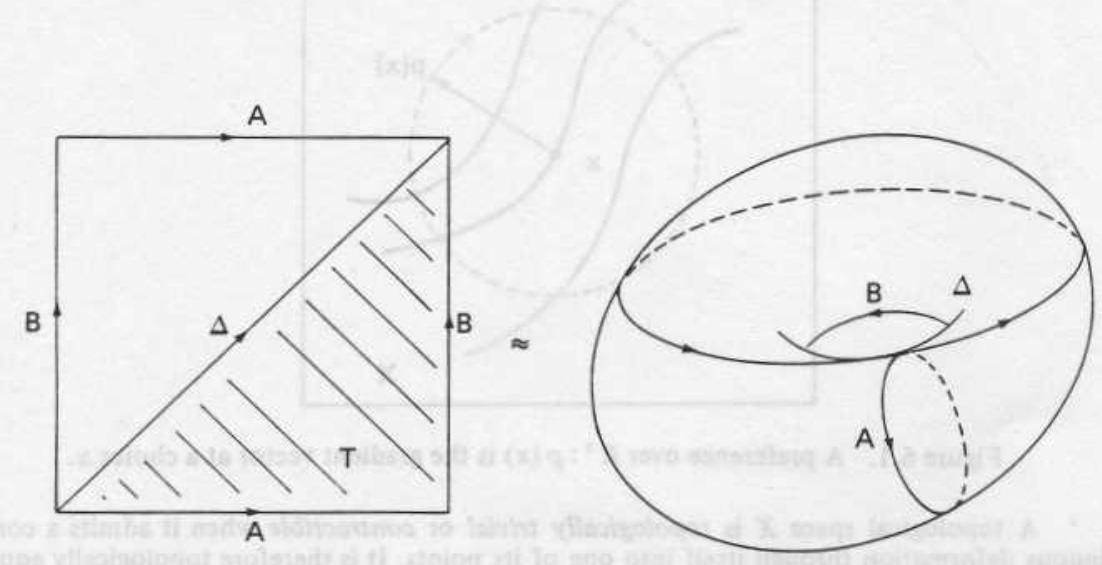

Figure 6.2. The space of profiles of linear preferences on $R^{2}$, with two voters. 
(1979b), in this particular case the space of preferences $P=S^{1}$, the one-dimensional circle in $R^{2}$. The space of profiles of two voters is thus

$$
P \times P=S^{1} \times S^{1},
$$

called also the two-dimensional torus (see fig. 6.2).

In what follows $D$ will represent the unit disk, $D=\left\{\left(x, y: x^{2}+y^{2} \leqslant 1\right)\right\}$, and $\partial D$ its boundary. The circle $P \times P=S^{1} \times S^{1}$ is equivalent to a square in which the points on opposite sides have been identified, as in the right-hand side of fig. 6.2. $A$ is the set $\left\{\left(p, p_{0}\right), p \in S^{1}\right\}$, and $B=\left\{\left(p_{0}, p\right), p \in S^{1}\right\}$. The boundary of the triangle $T$ is the union of the diagonal $\Delta=\left\{\left(p_{1}, p_{2}\right): p_{1}=p_{2}\right\}$ with $A$ and $B$, i.e. $\partial T=\Delta \cup A \cup B$. This can also be represented by three circles joined at one common point, as in fig. 6.3 .

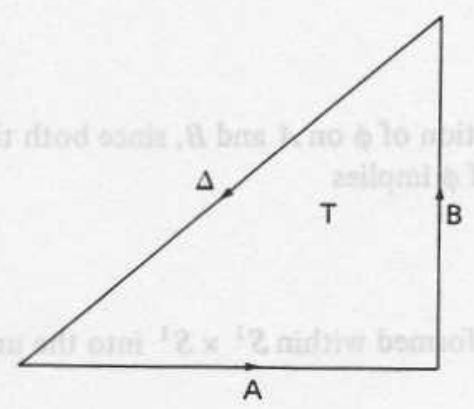

A

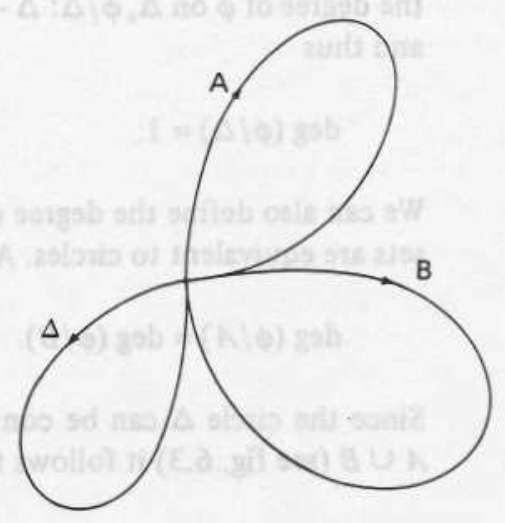

Figure 6.3

We need a definition from algebraic topology. The degree of a continuous map $f: S^{1} \rightarrow S^{1}$, denoted $\operatorname{deg}(f)$, can be described as the number of times $f\left(S^{1}\right)$ "covers" $S^{1}$ with the same orientation. For instance, if $f$ is the identity map on $S^{1}$, then $\operatorname{deg}(f)=1$; if $f(p)=2 p$ ( $p$ in radians), then $\operatorname{deg}(f)=2$; if $f\left(S^{1}\right)$ does not cover $S^{1}$ then $\operatorname{deg}(f)=0$. If a function $f$ changes orientation (e.g. $\left.f(x)=-x\right)$, then $\operatorname{deg}(f)$ is negative. See Spanier (1966) for a complete definition of "degree". 


\subsection{Social choice paradoxes and fixed points theorems}

The following theorem is valid for all preferences in $P$ (not necessarily linear), any finite number of voters, and any finite dimensional space of choices; the proof of the general case is in Chichilnisky (1980d):

Theorem 1. There is no continuous, anonymous social choice rule

$$
\phi: P^{2} \rightarrow P
$$

that respects unanimity.

In the particular case considered here, a simple geometrical proof can be given. Let $\Delta$ be the diagonal. Then $\Delta$ is equivalent to the circle $S^{1}$, and we can define the degree of $\phi$ on $\Delta, \phi / \Delta: \Delta \rightarrow S^{1}$. If $\phi$ respects unanimity, then $\phi(p, p)=p \forall p$, and thus

$$
\operatorname{deg}(\phi / \Delta)=1 .
$$

We can also define the degree of the restriction of $\phi$ on $A$ and $B$, since both these sets are equivalent to circles. Anonymity of $\phi$ implies

$$
\operatorname{deg}(\phi / A)=\operatorname{deg}(\phi / B) .
$$

Since the circle $\Delta$ can be continuously deformed within $S^{1} \times S^{1}$ into the union $A \cup B$ (see fig. 6.3) it follows that

$$
\operatorname{deg}(\phi / \Delta)=\operatorname{deg} \phi /(A \cup B) .
$$

On the other hand, the degree of $\phi$ on $A \cup B$ is the sum of the degree of $\phi$ on $A$ and on $B$ :

$$
\operatorname{deg} \phi /(A \cup B)=\operatorname{deg}(\phi / A)+\operatorname{deg}(\phi / B) .
$$

From (1)-(4) we obtain a contradiction because these equations imply that 1 is an even number. Thus, no continuous rule respecting unanimity can be anonymous.

Remark. The above result can be extended to preferences that admit satiation; see, for example, Chichilnisky (1982a). It should be pointed out that the linear 
preference whose gradient is zero (which is not considered here as a possible social outcome) is not merely a preference admitting satiation, but rather the preference which is indifferent among all possible choices. Clearly, such a social choice outcome would leave the social choice problem unresolved.

We now discuss briefly the connection between the paradox and fixed point theorems. The following result is proven in Chichilnisky (1979b).

Theorem 2. The existence of a continuous anonymous social rule respecting unanimity (as in theorem 1) is equivalent to the existence of a continuous function from the disk $D$ into itself, without any fixed point.

The idea underlying this result is simple. One proves that the paradox of theorem 1 is equivalent to that of extending a continuous function $g$ defined on the boundary of the disk $\partial D, g: \partial D \rightarrow \partial D$, into another function $f$ from all of the disk $D$ into $\partial D, f: D \rightarrow \partial D . f$ is called an extension of $g$ because it coincides with $g$ in their common domain, i.e. the following diagram commutes:

inclusion

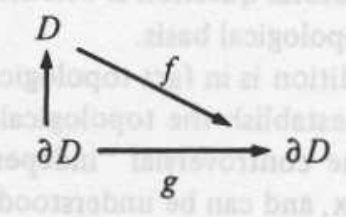

The equivalence between the paradox and the extension problem of (5) can be explained as follows. If $T$ is the triangle in $S^{1} \times S^{1}$ indicated in fig. 6.3, its interior $\stackrel{T}{T}$ is equivalent to the interior of disk $D$, and the frontier $\partial D$ corresponds to $\triangle \cup A \cup B$. Note that on the set $T$, the conditions on the rule $\phi$ are only restrictions of the behaviour of $\phi$ on $\Delta$, on $A$ and on $B$ : anonymity requires that $\phi / A \equiv \phi / B$, and unanimity is $\phi / \Delta=$ id $\Delta$.

One sees immediately that a continuous anonymous map respecting unanimity always exists on $\partial T$; for example:

$$
\begin{aligned}
& g / \Delta(p, p)=p, \quad \forall p \in S^{1}, \\
& g / A \equiv p_{0} \quad \text { and } \quad g / B \equiv p_{0},
\end{aligned}
$$

where $\triangle \cap A \cap B=\left(p_{0}, p_{0}\right)$. Therefore the paradox could be solved if such a function $g: \partial T \rightarrow S^{1}$ can be extended to another $f: T \rightarrow S^{1}$. Since $\partial T \cong \partial D$ and $S^{1} \cong \partial D$, the problem is equivalent to the extension of $g: \partial D \rightarrow \partial D$ into $f: D \rightarrow$ $\partial D$, as in diagram of (5). But such an extension exists if and only if $\operatorname{deg}(g / \partial D)$ 
$=0$. Since this latter statement is equivalent to Brouwer's fixed point theorem, see Chichilnisky (1979b), this explains the connection between a fixed point theorem and the paradox.

\subsection{Topological equivalence of the Pareto condition and the existence of a dictator}

Theorems 1 and 2 show that the paradox appears because the seemingly natural properties of anonymity and respect of unanimity are in contradiction with each other. These properties are obviously related, but are different, from two axioms that are the basis of Arrow's theorem: non-dictatorship and the Pareto condition. However, the two sets of conditions (Arrow's and ours) are not comparable, because Pareto is stronger than respect of unanimity, while on the other hand anonymity is stronger than non-dictatorship. In addition, Arrow imposes another, somewhat controversial, axiom: the independence from irrelevant alternatives, while here continuity is required. A natural question is whether, despite the differences, Arrow's paradox has also a topological basis.

We now show that the Pareto condition is in fact topologically equivalent to the existence of a dictator, and thus establish the topological basis of Arrow's theorem. This also establishes that the controversial "independence" axiom is not needed intrinsically for his paradox, and can be understood as a device to reduce the problem to the study of a finite number of choices (indeed, three choices as in the Condorcet paradox).

A comment about the domain of preferences may be useful here. While Arrow's paradox appears to be valid in principle with monotone preferences (while our theorem requires that gradients may vary in all directions) in fact this is not so. This is because the axiom of independence in Arrow's work reduces effectively the problem to preferences over three choices only. Over these three choices, say $a, b$, and $c$, in order to obtain his paradox, Arrow must allow for "Condorcet triples", i.e. three preferences ranking these choices as $(a, b, c),(c, a, b)$, and $(b, c, a)$, respectively. Obviously, no reasonable definition of monotonicity can be given that makes such Condorcet triples into a set of monotone preferences. Therefore, Arrow's theorem requires lack of monotonicity as well.

In what follows the results are a special case of the results in Chichilnisky (1982b) that prove the topological equivalence of the Pareto condition and the existence of a dictator, for any (finite) dimension of the choice space and any number of voters, using algebraic topology tools.

The following is a proof for a special case, which admits a geometric interpretation. A rule $\phi$ is said to be topologically equivalent to another $\widetilde{\phi}$ when there 
exists a continuous deformation of $\phi$ into $\tilde{\phi}$, i.e. a continuous function

$$
\Pi:(P \times P) \times[0,1] \rightarrow P \text {, }
$$

such that

$$
\begin{array}{lll}
\Pi\left(p_{1}, p_{2}, 0\right)=\phi\left(p_{1}, p_{2}\right), & \forall p_{1}, p_{2} \text { in } P x P, \\
\Pi\left(p_{1}, p_{2}, 1\right)=\widetilde{\phi}\left(p_{1}, p_{2}\right), & \forall p_{1}, p_{2} \text { in PxP. }
\end{array}
$$

We now examine geometrically the Pareto condition. If $p_{1}$ and $p_{2}$ are two preferences in $P$, the set of choices that both $p_{1}$ and $p_{2}$ prefer to $x$ is the "dual cone"

$$
E=\left\{q:\left(q \cdot p_{1}\right) \geqslant 0 \text { and }\left(q \cdot p_{2}\right) \geqslant 0\right\} \text {. }
$$

The Pareto condition requires that $\phi\left(p_{1}, p_{2}\right)$ prefers also all such choices to $x$, i.e.

$$
\phi\left(p_{1}, p_{2}\right) \in\{q:(q, r) \geqslant 0, \forall r \in E\} .
$$

This can only happen if $\phi\left(p_{1}, p_{2}\right)$ is in the "dual" of the "dual cone" $E$, i.e. the cone generated by $\left(p_{1}, p_{2}\right)$ denoted $C\left(p_{1}, p_{2}\right)$ (see fig. 6.4).

We can also show that if $\phi$ is Pareto, then $\operatorname{deg}(\phi / A)$ and $\operatorname{deg}(\phi / B)$ must be positive and at most 1 . This is because as $p$ varies over $S^{1}$, the outcome $\phi\left(p_{0}, p\right)$ must be in the cone $C\left(p_{0}, p\right)$. In particular, $\phi\left(p_{0}, p\right)$ cannot cover all of $S^{1}$ unless $p$ has covered it. The proof of the next theorem will also show that a Pareto rule $\phi$ cannot have degree one simultaneously on $A$ and on $B$. This is because a

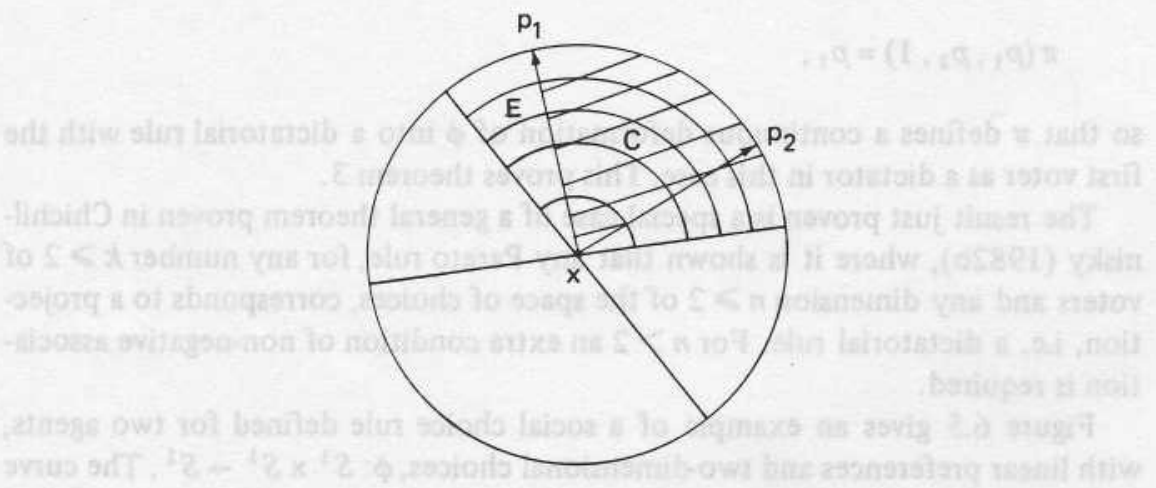

Figure 6.4. The set shaded in circular lines is the dual cone $E$ corresponding to $\left(p_{1}, p_{2}\right)$. The double shaded set is the cone generated by $\left(p_{1}, p_{2}\right), C\left(p_{1}, p_{2}\right)$. 
Pareto rule is shown in the next theorem to be topologically equivalent to a dictatorial rule, and the degree of a map only depends on its topological equivalence class. Thus, since the degree of a dictatorial (i.e. a projection) map $\Pi: S^{1} \times S^{1} \rightarrow$ $S^{1}$ is 1 on one of the sets $A$ or $B$, and 0 on the other, the same must be true of a Pareto rule.

Theorem 3. If $\phi: P \times P \rightarrow P$ is Pareto, $\phi$ is topologically equivalent to a dictatorial rule.

Proof. Let $p_{1}$ and $-p_{1}$ be two diametrically opposed vectors in $S^{1}$. The Pareto condition and the continuity of $\phi$ together imply that either $\phi\left(p_{1},-p_{1}\right)=p_{1}$, or else $\phi\left(p_{1},-p_{1}\right)=-p_{1}$. Without loss of generality, assume $\phi=\left(p_{1},-p_{1}\right)=$ $p_{1}$. By continuity, we then have $\phi\left(p_{2},-p_{2}\right)=p_{2}$ for all $p_{2}$ in $S^{1}$. Moreover, $\phi\left(p_{1}, q\right)$ must be different from $-p_{1}$ for all $q$ in $S^{1}$ with $q \neq-p_{1}$, since $\phi\left(p_{1}\right.$, $q)$ must belong to the cone $C\left(p_{1}, q\right)$. Thus, for all $p_{1} \in S^{1}, \phi\left(p_{1}, q\right) \neq-p_{1}, \forall q$ in $S^{1}$. Let $\pi: P \times P \times[0,1] \rightarrow P$ be defined by

$$
\pi\left(p_{1}, p_{2}, t\right)=\frac{t\left(p_{1}\right)+(1-t) \phi\left(p_{1}, p_{2}\right)}{\left\|t\left(p_{1}\right)+(1-t) \phi\left(p_{1}, p_{2}\right)\right\|} .
$$

In view of the above remarks, the denominator of this expression never vanishes, and therefore $\pi$ is a continuous map. In addition:

$$
\pi\left(p_{1}, p_{2}, 0\right)=\phi\left(p_{1}, p_{2}\right)
$$

and

$$
\pi\left(p_{1}, p_{2}, 1\right)=p_{1}
$$

so that $\pi$ defines a continuous deformation of $\phi$ into a dictatorial rule with the first voter as a dictator in this case. This proves theorem 3.

The result just proven is a special case of a general theorem proven in Chichilnisky $(1982 b)$, where it is shown that any Pareto rule, for any number $k \geqslant 2$ of voters and any dimension $n \geqslant 2$ of the space of choices, corresponds to a projection, i.e. a dictatorial rule. For $n>2$ an extra condition of non-negative association is required.

Figure 6.5 gives an example of a social choice rule defined for two agents, with linear preferences and two-dimensional choices, $\phi: S^{1} \times S^{1} \rightarrow S^{1}$. The curve drawn in the left-hand figure is the set $\left\{\phi^{-1}(\bar{p})\right\}$, the inverse image under $\phi$ of an outcome $\bar{p}$ in $S^{1}$. Under the assumption of smoothness and regularity, $\left\{\phi^{-1}\right.$ 

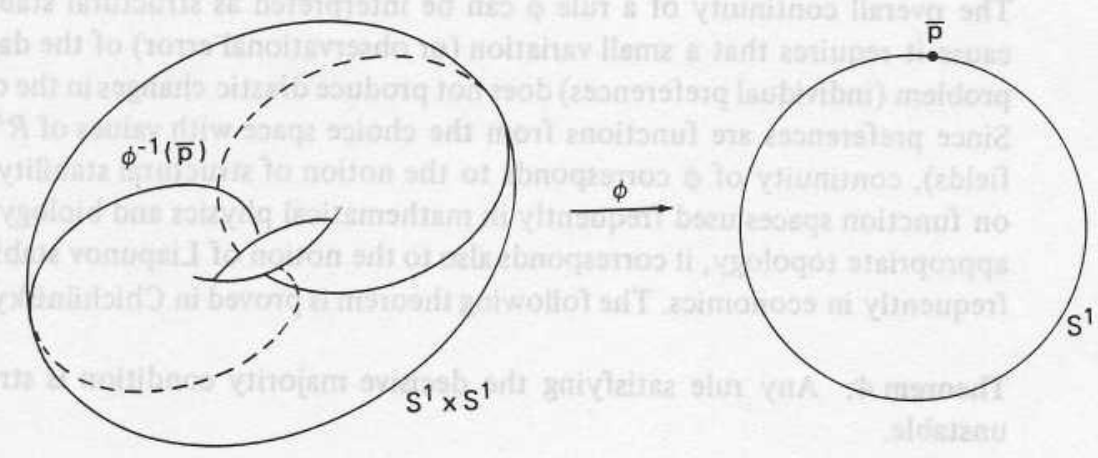

Figure 6.5

$(\bar{p})\}$ is a one-dimensional manifold, by the global version of the implicit function theorem. Note that each point $\bar{p}$ in $S^{1}$ is attained exactly twice (with the same orientation) as a value of the map $\phi$ restricted to either set $A$ or $B$. Therefore degree $\phi / A=2$, and thus $\phi$ cannot be Pareto in view of the above; of course, $\phi$ cannot be deformed into a dictatorial rule either.

\subsection{Decisive majority rules}

A social choice rule is said to satisfy a decisive majority condition if in certain particular cases the outcome chosen coincides with that of the majority, namely in those cases when the voters can be divided into two groups, within each graph voters have identical preferences, and across groups they have opposite preferences. Formally, $\phi$ is a decisive majority rule if for all profile $\left(p_{1}, p_{2}, \ldots, p_{k}\right)$ such that $p_{i}=p$ or $p_{i}=-p, \forall i=1, \ldots, k$, then

$$
\phi=\left(p_{1}, p_{2}, \ldots, p_{n}\right)=p
$$

if the number of voters with preference $p$ exceed those with preference $-p$.

A majority rule clearly satisfies a decisive majority condition, but the converse is obviously not true. This is because the decisive majority condition is only binding in the particular case in which the voters can be divided into two totally internally homogeneous groups for all possible choices, and the two groups are completely opposed to each other, also for all possible choices. Therefore, a decisive majority condition is much weaker than a majority condition. 
In our next result we explore the structural stability of decisive majority rules. The overall continuity of a rule $\phi$ can be interpreted as structural stability, because it requires that a small variation (or observational error) of the data of the problem (individual preferences) does not produce drastic changes in the outcome. Since preferences are functions from the choice space with values of $R^{n}$ (vector fields), continuity of $\phi$ corresponds to the notion of structural stability of maps on function spaces used frequently in mathematical physics and biology; with an appropriate topology, it corresponds also to the notion of Liapunov stability used frequently in economics. The following theorem is proved in Chichilnisky (1981):

Theorem 4. Any rule satisfying the decisive majority condition is structurally unstable.

The following is an immediate corollary:

Corollary 5. Majority rules are structurally unstable.

\subsection{Intensity of preference and Von Neumann-Morgenstern utilities}

The preferences considered until now have been ordinal, and the intensity of preferences was not recorded. Such preferences rank choices in a given order, but do not measure the difference in the intensity of preferences between, for example, choices $a$ and $b$, and between $c$ and another choice $d . a$ may be preferred to $b$ and $c$ to $d$, but one may want to consider whether $a$ is preferred to $b$ more than $c$ is preferred to $d$.

This is precisely formalized in the notion of cardinal preferences. Cardinal preferences are given by utility representations which are invariant under, and only under, linear positive transformations. By comparison, the invariance required of ordinal preferences is far greater, involving all monotonic transformation (not just linear ones). In the case of choice under uncertainty it is shown in Chichilnisky (1980b) that with this definition, cardinal preferences coincide with Von Neumann-Morgenstern utilities. The result obtained for the aggregation of cardinal preferences are thus applied directly to the aggregation of Von Neumann -Morgenstern utilities.

In contrast to all the previous results, we now consider discrete sets of choices, either finite or numerable. In the finite case the choice space is therefore $X=$ $\left\{x_{1}, \ldots, x_{n}\right\}$, and we have shown in Chichilnisky (1980b) that the set of (nonzero) cardinal preferences can be represented by 


$$
R=\left\{\left(p_{1}, \ldots, p_{n}\right) \in R^{n+}: 0 \leqslant p_{i} \leqslant 1 \forall i ; p_{j}=0 \text { and } p_{k}=1 \text { for some } j, k\right\} \text {. }
$$

Each $p_{i}$ describes the utility value of choice $x_{i}$. In addition, we also consider the null preference, which is indifferent among all choices, represented by the vector

$$
\{0\}=(0, \ldots, 0) \text {. }
$$

The space of all cardinal preferences is therefore

$$
Q=R \cup\{0\}
$$

This space has two connected components $R$ and $\{0\}$. A similar definition can be given for the case where there are infinite (countably many) choices. In this case the space of cardinal preferences is a subspace of Banach space of infinite sequences (Chichilnisky, 1980b).

In what follows we consider rules for aggregation of cardinal preferences: with $k$ voters, they are functions of the form

$$
\phi: Q^{k} \rightarrow Q
$$

The social preference which is indifferent among all choices, i.e. $\{0\}$, is a permissible outcome.

Theorem 6. If the space $X$ of choices is finite, then there exists no continuous aggregation rule for cardinal preferences $\phi: Q^{k} \rightarrow Q$ that respects unanimity and anonymity. This result is also valid for the aggregation of Von Neumann-Morgenstern utility functions.

This result is proved in Chichilnisky (1980b) through the study of the topological structure of spaces of cardinal preferences and spaces of Von NeumannMorgenstern utilities. In particular, the following lemma is obtained.

Lemma 7. With a finite set of alternatives, the space of Von Neumann-Morgenstern utilities is not contractible.

It turns out that the topology of the spaces of cardinal preferences changes drastically with infinitely many alternatives. In this case the spaces of cardinal preferences become topologically trivial or contractible, and we have the following: 
Theorem 8. With an infinite (countable) number of alternatives, there exists a continuous anonymous aggregation rule for cardinal preferences $\phi: Q^{k} \rightarrow Q$ respecting unanimity, and it is a deformation of a Bergson-Samuelson welfare function. However, this function is the limit of dictatorial rules on finite subsets of choices. The same result applies to the aggregation of Von Neumann-Morgenstern utilities with countably many alternatives. (See Chichilnisky, 1980b.)

The above results show the difference between spaces of cardinal and ordinal preferences. For ordinal preferences, we obtained non-existence results when the choice space $X$ is infinite, such as the cube in Euclidean space of the previous sections. With cardinal preferences, and Von Neumann-Morgenstern utilities, we obtain instead non-existence results with finitely many choices, and existence results with infinitely many choices. One reason for this difference is that spaces of ordinal preferences are finite dimensional even when the choices are infinitely many; by contrast, cardinal preferences and Von Neumann-Morgenstern utilities define infinite dimensional spaces whenever the set of choices is infinite. The following subsection will explore another characteristic of infinity, namely the case of large economies or economies with infinite populations. Recall that all the results until now have referred to finitely many voters only. The following results study finite dimensional choice spaces but infinitely large populations.

\subsection{Social choice in large economies}

As seen above, infinite dimensionality produces significant differences in the results. The reason is that the topology of infinite dimensional spaces is rather different from that of Euclidean spaces. A particular characteristic that affects our results is that while in Euclidean spaces the circle $S^{1}$ and in general any (hollow) sphere is not contractible or topologically trivial, in infinite dimensional Banach spaces show hollow spheres are contractible (see, for example, Kuiper, 1971).

Theorem 8. Let $P^{\infty}=\mathrm{X}_{i=1}^{\infty} P$ be the space of profiles of a countable number of agents and $P$ the space of ordinal preferences defined on $n$-dimensional Euclidean choice space. Then the rule $\phi\left(p_{1}, \ldots, p_{k}, \ldots\right)=\lim _{k \rightarrow \infty} p_{k}$ is continuous, Pareto, and non-dictatorial (see Chichilnisky and Heal, 1979b).

A note on the concept of limit: if the sequence of vectors $\left\{p_{i}(x)\right\}$ has a standard limit in Euclidean space for all choices $x$, then the outcome of the rule coincides with this limit. Otherwise, the limit is defined by means of a free ultrafilter of the integers $\{1,2, \ldots, k, \ldots\}$. (See definition in Chichilnisky and Heal, 
1979b.) It can be seen that the rule $\phi$ is well defined, and continuous with respect to the standard product topology of $P^{\infty}$. It is worth noting that the literature in this area succeeded previously in proving the existence of Pareto, non-dictatorial rules (see, for example, Brown, 1974), but not the existence of such continuous rules. An example is provided in fig. 6.6. In this figure, $p(x)=\lim _{i} p_{i}(x)$ is by definition the image $\phi$ of the sequence of vectors $\left\{p_{1}(x), p_{2}(x), \ldots, p_{n}(x), \ldots\right\}$,

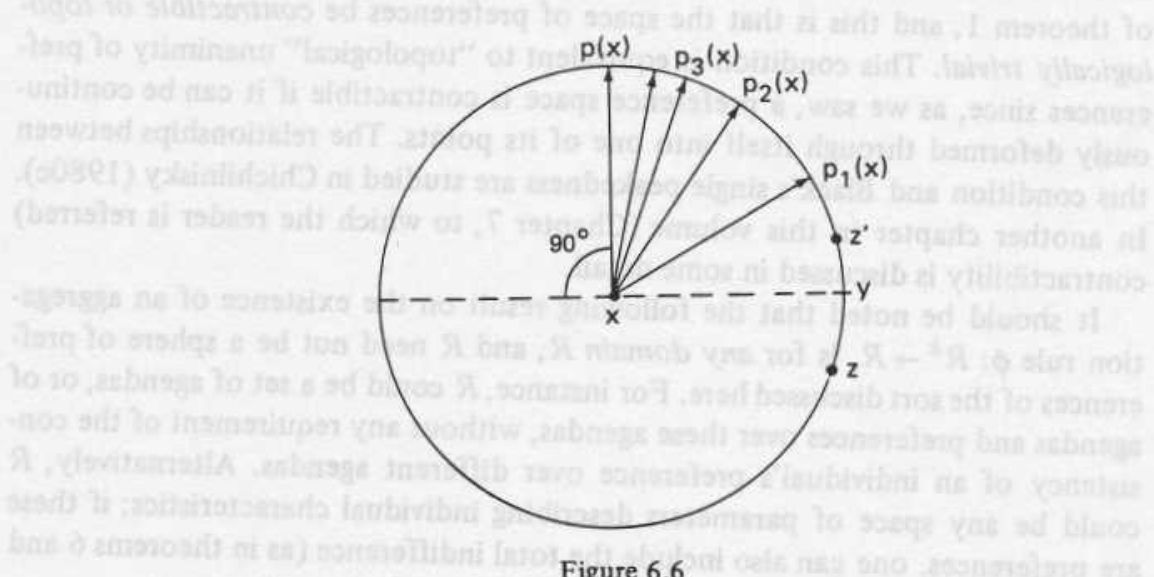

Figure 6.6

as in theorem 8 above. In contrast, in Brown (1974) the "winning coalitions" that decide the outcome are the subsets of a free ultrafilter $F$ of the integers $N$. If $F$ is the free ultrafilter over $N$ consisting of the complements of finite subsets of $N$, then the collective preference according to Brown (1974) prefers $y$ strictly to $x$ while $\lim _{i} p_{i}(x)$ (our definition) is indifferent between $y$ and $x$. Note that the rules of Brown (1974) are in effect discontinuous, because they prefer $x$ strictly to any point $z$ in the circle to the right of $y$, while they prefer strictly any point $z^{\prime}$ to the left of $y$ in the circle, and $y$, to $x$.

\subsection{Necessary and sufficient conditions for a resolution of the social choice paradox}

The results discussed above show that without restrictions on preferences it is impossible to eliminate the aggregation paradox. A traditional approach has been to study conditions to eliminate the paradox, these being given as restrictions on the domain of individual preferences. The first such conditions were given by Black (1948) who introduced the notion of "single peakedness", meaning that one can order the (finite) set of choices in a line in such a way that each agent 
has a unique alternative who he or she prefers best (called peak), and that to the right and to the left of this alternative choices become progressively less desirable as they are further away from the peak. This remains the only sufficient condition which is generally accepted and utilized to solve the paradox (see also Sen, 1970).

In another paper Chichilnisky and Heal (1979a) found that there exists a domain restriction which is both necessary and sufficient to eliminate the paradox of theorem 1, and this is that the space of preferences be contractible or topologically trivial. This condition is equivalent to "topological" unanimity of preferences since, as we saw, a preference space is contractible if it can be continuously deformed through itself into one of its points. The relationships between this condition and Black's single peakedness are studied in Chichilnisky (1980c). In another chapter in this volume (Chapter 7, to which the reader is referred) contractibility is discussed in some detail.

It should be noted that the following result on the existence of an aggregation rule $\phi: R^{k} \rightarrow R$, is for any domain $R$, and $R$ need not be a sphere of preferences of the sort discussed here. For instance, $R$ could be a set of agendas, or of agendas and preferences over these agendas, without any requirement of the consistency of an individual's preference over different agendas. Alternatively, $R$ could be any space of parameters describing individual characteristics; if these are preferences, one can also include the total indifference (as in theorems 6 and 7) as well as incomplete preferences on a Euclidean choice space.

Theorem 10. Let $R$ be a manifold representing individual characteristics. Then a continuous rule $\phi: R^{k} \rightarrow R$ that respects anonymity and unanimity exists if and only if $R$ is contractible.

For a proof see Chichilnisky and Heal (1979a). It is of interest to note that if such a rule exists, then it is topologically equivalent to a Bergsonian welfare function:

Theorem 11. If the dimension of $R$ exceeds 5 , and the boundary $\partial R$ is simply connected, ${ }^{2}$ then any continuous rule $\phi: R^{k} \rightarrow R$ which is anonymous and respects unanimity is topologically equivalent to the rule

$$
\phi\left(p_{1}, \ldots, p_{k}\right)=\frac{1}{k} \sum_{i=1}^{k} p_{k} .
$$

This result follows from theorem 10. This is because if such a $\phi$ exists, then $R$

2 I.e. the first homotopy group $\pi_{1}(\partial R)$ is zero; see Spanier (1966) for a description of homotopy groups. 
must be contractible, and when $R$ is contractible any map $\phi: R^{k} \rightarrow R$ will be a deformation of a convex addition rule, because by the proof of the Poincaré conjecture (under the conditions) $R$ is deformable into a convex set.

Finally we mention the following:

Theorem 12. A continuous deformation of the (restricted) space of preferences $R$ satisfies the "single peakedness" condition if and only if $R$ is contractible, i.e. if and only if a continuous anonymous rule $\phi: R^{k} \rightarrow R$ respecting unanimity exists.

For a proof, see Chichilnisky (1980c).

\section{Game theory}

The basic mathematical structure of game theory is rather similar to that of social choice theory. A game consists of two objects: a map that associates an outcome to each $k$-tuple of individual strategies, denoted

$$
g: S^{k} \rightarrow A,
$$

where $S$ is the space of messages or strategies and $A$ the space of outcomes; and of a $k$-tuple of individual preferences over outcomes. ${ }^{3}$ The basic properties of such a game form $g$ are the subject of game theory, much the same way that the study of the aggregation maps $\phi: P^{k} \rightarrow P$ are those of social theory. In both cases one is concerned with maps from a product space to another space.

An initial difference between these two fields arises from the fact that the players of the game are aware of the game form $g$, and therefore choose their messages in $S$ strategically. In social choice theory, instead, the properties of the map $\phi$ are the concern of the planner only (individuals are assumed simply to announce their preferences) and the relevant properties of a social choice rule $\phi$ are those of justice. In game theory, instead, the properties of a game form $g$ are of direct interest to the players, and they choose their strategies in order to maximize the value of the outcome, according to their own preferences.

A direct link between the two problems arises with the issue of manipulability of social choice rules. In this case the agents are assumed to know the aggregation rule $\phi$, and to choose strategically the preference they announce to the planner in order to influence the outcome in their favour. For instance, with an ade-

3 The function $g$ is called a game form. 
quate definition of distance between preferences, the goal of the manipulation of messages would be to obtain a collective preference as close as possible to one's own. This section studies such problems. A large body of literature exists in this area and is not reviewed here. For references see, for example, Chichilnisky (1982c) and Chichilnisky and Heal (1982), and the original work of Vickrey (1960), Gibbard (1973) and Satterthwaite (1975).

An obvious technical difference between social choice and game theories is that the space of outcomes $A$ can be rather different from the space of messages. Since an aggregation rule is a map $\phi: P^{k} \rightarrow P$, while a game form is $g: S^{k} \rightarrow A$, where in general $A \neq S$, the two problems have a somewhat different structure. This difference does not appear to be a fundamental one, because the properties of a map defined on the $k$-product of a space with itself with values in the same space, are related to the property of the product space into other spaces that are significantly simpler, such as outcome spaces generally are.

A second difference appears because of the equilibrium concepts of game theory. This displaces the topology structure of the problem, towards the geometry or differential topology, since notions of equilibrium are generally based on maximization.

\section{Manipulation of games}

As will be seen in what follows, the manipulation of a social choice rule, or more generally of a game, is in many cases a topological problem, while the existence and properties of an equilibrium are better studied instead by geometric or differentiable means. Under certain conditions, however, the topological analysis will serve also to establish the existence and properties of the equilibrium of a game. In particular, the following theorem will show that certain games have a Nash equilibrium only if they are "unfair" in the sense that they can be manipulated by one player more than by others and this is a topological property. Thus "more cooperative" solutions than Nash equilibrium are needed in order to secure the equity of games.

Within the games studied below, the players' strategies consist of announcing a preference, and the outcomes are aggregate preferences. The player wishes, ideally, to attain an outcome as close as possible to his or her true preference, so that preference over outcomes are given by distance functions.

If one considers more general outcome spaces, the results can be extended by noting, for instance, that a social choice in $R^{n}$ may be obtained from the maximization of social preferences on $R^{n}$. Therefore, one can construct games $h$ where outcomes are arbitrary vectors in $R^{n}$ deriving them from the games $g$ constructed here by the rule 


$$
h=\mathrm{M} \circ g \text {, }
$$

or

$$
h=P^{k} \stackrel{\mathrm{g}}{\rightarrow} P \stackrel{\mathrm{M}}{\rightarrow} A,
$$

where $\mathrm{M}$ denotes the maximization operator.

The following result is proven in Chichilnisky (1979a) utilizing homotopy theory:

Theorem 13. If $g: P^{k} \rightarrow P$ is a continuous game respecting unanimity, then it is either dictatorial, or else there exists a player that can obtain any outcome he or she desires by announcing a false preference. In addition, if $g$ is Pareto such a manipulative player is unique.

Formally, we say that a player (say the first) is a strong manipulator if $\forall p_{1}^{*} \in P$, and all $p_{2} \in P, \exists p_{1}=p_{1}\left(p_{2}\right)$ such that

$$
\phi\left(p_{1}, p_{2}\right)=p_{1}^{*}
$$

Theorem 13 establishes therefore the existence of a strong manipulator for continuous games which respect unanimity.

Proof of theorem 13 in a special case. Consider now, as before, the special case where there are two players with linear preferences, so that

$$
g: S^{1} \times S^{1} \rightarrow S^{1} \text {. }
$$

As seen in theorem 1 , if $g$ respects unanimity,

$$
\operatorname{deg}(g / \Delta)=1
$$

Since $\operatorname{deg}(g /(A \cup B)=\operatorname{deg}(g / \Delta)=1$, and $\operatorname{deg} g /(A \cup B)=\operatorname{deg} g / A+\operatorname{deg} g / B$, it follows that either $\operatorname{deg}(g / A) \neq 0$, or $\operatorname{deg}(g / B) \neq 0$. Assume, without loss of generality, that $\operatorname{deg}(g / A) \neq 0$. Then the first player is a strong manipulator, because the image of $A$ under $g, g / A(A)$, must cover $S^{1}$, otherwise deg $(g / A)$ would be zero. This means that when the second player states $p_{0}$, there exists some $p_{1}=p_{1}\left(p_{0}\right)$ such that

$$
g\left(p_{1}, p_{0}\right)=p_{1}^{*},
$$


for any $p_{1}^{*}$ in $S^{1}$. Since it can be seen that $\operatorname{deg}(g / A) \neq 0$ implies that $\operatorname{deg}\left(g / A^{\prime}\right)$ $\neq 0$ for any set $A^{\prime}=\left\{(p, q): q=q_{0}, p \in S^{1}\right\}$, then the first part of the theorem is proven. To prove the second part, note that when $g$ is Pareto, as seen above, then

$$
0 \leqslant \operatorname{deg}(g / A) \leqslant 1
$$

and

$$
0 \leqslant \operatorname{deg}(g / B) \leqslant 1
$$

therefore $\operatorname{deg}(g / A)+\operatorname{deg}(g / B)=1$ implies the existence of a unique manipulator. When $g$ is not dictatorial, then the manipulator must lie.

One can now introduce the notion of equity in such a game. Assume $g:\left(S^{1}\right)^{2}$ $\rightarrow S^{1}$ is equitable, or fair when either both players can manipulate the game, or when neither of them can. An example of a game which has one strong manipulator is shown in fig. 6.7.

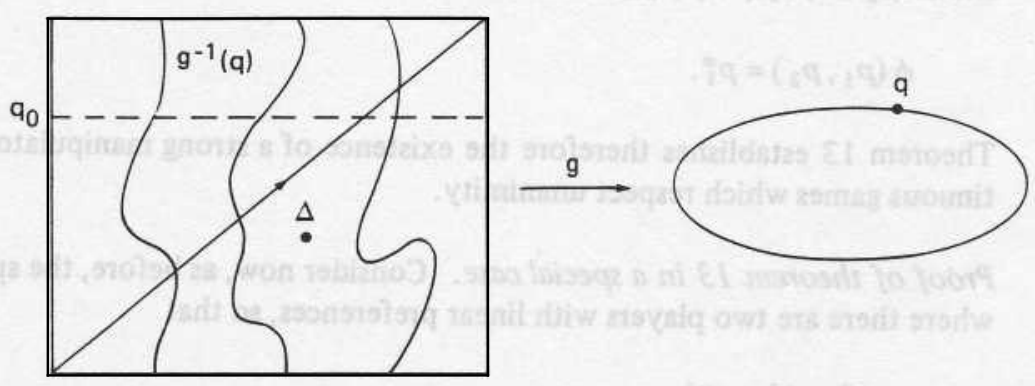

Figure 6.7. The curves in the left-hand diagram indicate three different hypersurfaces of the game $g$ : $S^{1} \times S^{1} \rightarrow S^{1}$. Since by unanimity $\operatorname{deg} g / \Delta=1$, then for any $q$ in $S^{1}, \phi^{-1}(q) \cap \Delta$ $=q$. Note that for all $q$ and any $q_{0}$, there is a $p$ such that $g\left(p, q_{0}\right)=q$.
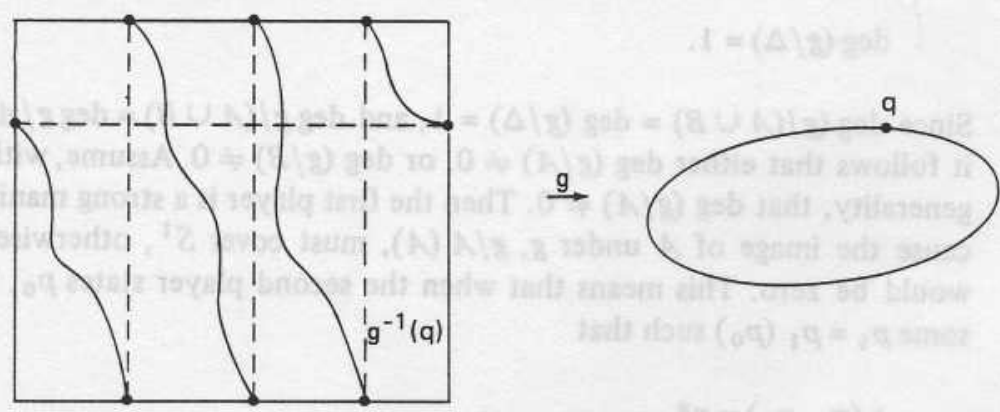

Figure 6.8. The union of the curves in the left-hand diagram represents one hypersurface of the game. This game has two manipulators: for any $q_{0}$ there is a $p_{1}$ such that $g\left(p_{1}, p_{0}\right)$ $=q$, and a $p_{2}$ such that $g\left(q_{0}, p_{2}\right)=q$. 
The game in fig. 6.7 is clearly not dictatorial. An example of a game with two manipulators is shown in fig. 6.8 .

Clearly, a game with two strong manipulators cannot have a Nash equilibrium when the two players have different "true" preferences, since each player can attain the most desirable outcome by an appropriate choice of strategy once the other player's announcement is known. The following theorem establishes the relationship between existence of Nash equilibria and fairness of games.

Theorem 14. A continuous game $g$ that satisfies unanimity has a Nash equilibrium only if it is unfair.

This is established by an application of theorem 13. The game is fair when there are no manipulators, or when both players can manipulate the game. The first case is eliminated by theorem 13 . In the second case there is no Nash equilibrium. Unfairness is therefore necessary for the existence of a Nash equilibrium.

We conclude this section with recent results on the existence and characterization of non-manipulable games. Clearly, if one wishes to preserve respect of unanimity, in order to prevent manipulability one must restrict the domain of individual preferences. This is the same procedure that was followed in the first part of this paper to find solutions to the social choice paradox.

The following results will therefore study games with arbitrary strategy and outcome spaces; these need not be preference spaces.

The spaces considered here are smooth manifolds $X$, of dimension at least 5 , and with simply connected boundaries (i.e. the first homotopy group $\Pi_{1}(\partial X)=0$ ). A straightforward game is one in which the announcement of an agent's true characteristic is a dominant strategy for each player. We consider here games which are onto, i.e. the image of $g: X^{k} \rightarrow X$ covers $X$.

Theorem 15. A continuous straightforward game $g: X^{k} \rightarrow X$ respecting anonymity exists if and only if $X$ is contractible.

For a proof see Chichilnisky (1982c). This last theorem shows the close link between the conditions needed to solve the social choice paradox (theorem 10) and those needed for the existence of straightforward games: the necessary and sufficient condition in both cases is contractibility, or topological triviality. Aggregation problems and manipulable games exist in those cases where the relevant spaces (of preferences, or strategies and outcomes, respectively) are topologically complex.

Consider now the case where choices are vectors in $R^{n}$, messages are single peaked preferences, of the form shown in fig. 6.9 , given by the distance function 


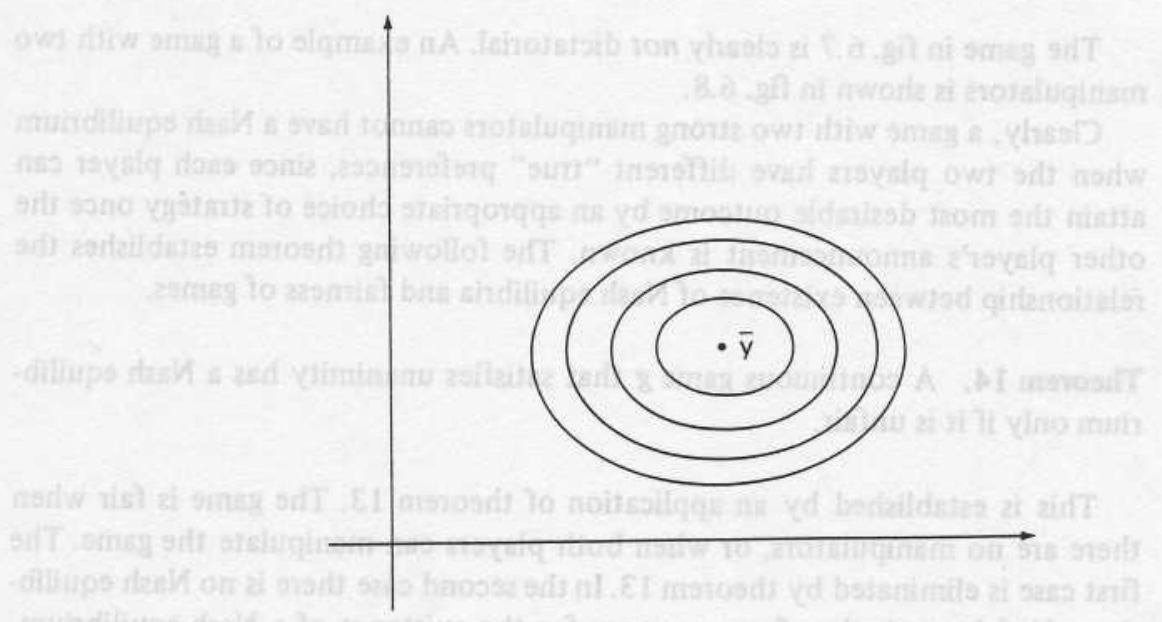

Figure 6.9. A single peaked preference on $R^{2}$ : the curves indicate the indifference surfaces.

$$
d(x, \bar{y})=\sum_{i=1}^{k} a_{i}\left(x_{i}-\bar{y}_{i}\right)^{2}, \quad a_{i}>0, \forall i=1, \ldots, n
$$

where $\bar{y}$ denotes the "bliss" point. Outcomes are vectors in $R^{n}$ as well. The space of messages in denoted $M$, and the space of outcomes $A$.

A function $g:\left(R^{n}\right)^{\kappa} \rightarrow R^{n}$ is said to be locally simple if it is continuous and separable, i.e.

$$
\begin{aligned}
& g\left(r_{1}^{1}, \ldots, r_{n}^{1}, r_{1}^{2}, \ldots, r_{n}^{2}, \ldots, r_{1}^{k}, \ldots, r_{n}^{k}\right) \\
& =g_{1}\left(r_{1}^{1}, \ldots, r_{n}^{1}\right), g_{2}\left(r_{1}^{2}, \ldots, r_{n}^{2}\right), \ldots, g_{k}\left(r_{1}^{k}, \ldots, r_{n}^{k}\right),
\end{aligned}
$$

and for almost all $\left(x_{1}, \ldots, x_{k}\right) \in\left(R^{n}\right)^{k}, g$ is either constant, or dictatorial (i.e. a projection) on some neighbourhood of $\left(x_{1}, \ldots, x_{k}\right)$ in $\left(R^{n}\right)^{k}$.

The following is a characterization of all straightforward games in this context.

Theorem 16. $g: M^{k} \rightarrow A$ is a straightforward game if and only if the function $g$ is locally simple. For a proof see Chichilnisky and Heal (1982).

Remark. Note that theorem 15 implies, in particular, that whenever $g$ is straightforward, then $g$ is continuous. 
For such straightforward games continuity is therefore a result rather than a condition.

A final result gives conditions for a game to be Nash implementable, i.e. for the truthful revelation of characteristics, when each player plays a Nash equilibrium strategy. Since straightforward games require a more stringent equilibrium concept, i.e. that truthful revelation is a dominant strategy. It may appear that one could obtain larger classes of Nash implementable than straightforward games; the following result shows that, at least for separable games, this is not the case:

Theorem 17. A separable regular game $g: M^{k} \rightarrow A$ is Nash implementable only if $g$ is locally simple. For a proof see Chichilnisky and Heal (1981).

Remark. The regularity condition requires that $g$ be smooth, and a transversality condition which is generally satisfied. See Chichilnisky and Heal (1981).

\section{References}

Arrow, K.J. (1951) Social Choice and Individual Values (Yale University Press).

Black, D. (1948) "On the Rationale of Group Decision Making", Journal of Political Economy.

Brown, D. (1974) "Aggregation of Preferences", Quarterly Journal of Economics.

Chichilnisky, G. (1976) "Manifolds of Preferences and Equilibria", Project for Efficiency of Decision Making in Economic Systems, Harvard University.

Chichilnisky, G. (1978) "Spaces of Economic Agents", Journal of Economic Theory.

Chichilnisky, G. (1979a) "A General Result on Strong Manipulability", The Economic Workshop, Columbia University.

Chichilnisky, G. (1979b) "On Fixed Points and Social Choice Paradoxes", Economic Letters.

Chichilnisky, G. (1980a) "Continuous Representation of Preferences", Review of Economic Studies.

Chichilnisky, G. (1980b) "Intensity of Preferences and von Neuman Morgenstern Utilities", Working Paper, University of Essex.

Chichilnisky, G. (1980c) "Single Peakedness and the Contractibility of Preference Spaces", Working Paper, University of Essex.

Chichilnisky, G. (1980d) "Social Choice and the Topology of Spaces of Preferences", Advances in Mathematics.

Chichilnisky, G. (1981) "The Structural Instability of Decisive Majority Rules", Journal of Mathematical Economics.

Chichilnisky, G. (1982a) "Social Aggregation Rules and Continuity", Quarterly Journal of Economics.

Chichilnisky, G. (1982b) "The Topological Equivalence of the Pareto Condition and the Existence of a Dictator", Journal of Mathematical Economics.

Chichilnisky, G. (1982c) "Incentive Compatible Games: A Characterization of Strategy and Outcome Spaces", Working Paper No. 139, Columbia University.

Chichilnisky, G. and G. Heal (1979a) "Necessary and Sufficient Conditions for a Resolution of the Social Choice Paradox", Journal of Economic Theory, in press. 
Chichilnisky, G. and G. Heal (1979b) "Social Choice with Infinite Population: Construction of a Rule and Impossibility Results", The Economic Workshop, Columbia University.

Chichilnisky, G. and G. Heal (1980) "Patterns of Power: Bargaining and Implementation in two person games", Discussion Paper, University of Essex.

Chichilnisky, G. and G. Heal (1981) "Incentive Compatibility and Local Simplicity", Working Paper, University of Essex.

Chichilnisky, G. and G. Heal (1982) "A Necessary and Sufficient Condition for Straight Forward Games", Working Paper No. 138, Columbia University.

Debreu, G. (1972) "Regular Economies", Econometrica.

Gibbard, A. (1973) "Manipulation of Voting Schemes: A General Result", Econometrica.

Kuiper, N.H. (1971) Variétés Hilbertiennes. Aspects Géométriques, Séminaire de mathématiques Supérieures (la Pressé de l'Université de Montréal).

Satterthwaite, M.A. (1975) "Strategy-Proofness and Arrow's Conditions: Existence and Correspondence Theorems for Voting Procedures and Social Welfare Functions", Journal of Economic Theory.

Sen, A.K. (1970) Collective Choice and Social Welfare (Holden-Day, San Francisco).

Spanier, E. (1966) Algebraic Topology (McGraw-Hill, New York).

Vickrey, W. (1960) "Utility, Strategy and Social Decision Rules", Quarterly Journal of Economics. 